\title{
Concepções das enfermeiras sobre assistência segura à mulher na gestação e parto
}

\author{
Nurses' conceptions of safe care for women during pregnancy and childbirth \\ Concepciones de las enfermeras sobre la atención segura de la mujer durante el embarazo y el \\ parto
}

Recebido: 26/02/2021 | Revisado: 05/03/2021 | Aceito: 12/03/2021 | Publicado: 19/03/2021

\author{
Deise Breder dos Santos Batista \\ ORCID: https://orcid.org/0000-0001-6530-2657 \\ Universidade do Estado do Rio de Janeiro, Brasil. \\ E-mail: deisebreder08@gmail.com \\ Juliana Cristina Nascimento Guimarães \\ ORCID: https://orcid.org/0000-0001-9819-1463 \\ Universidade do Estado do Rio de Janeiro, Brasil. \\ E-mail: julianacristinna3@gmail.com \\ Yasmin Miranda Alfredo \\ ORCID: https://orcid.org/0000-0002-1376-3141 \\ Universidade do Estado do Rio de Janeiro, Brasil. \\ E-mail: yasminalfredo67@gmail.com \\ Adriana Lenho de Figueiredo Pereira \\ ORCID: https://orcid.org/0000-0002-2563-6174 \\ Universidade do Estado do Rio de Janeiro, Brasil. \\ E-mail: adrianalenho.uerj@gmail.com
}

\begin{abstract}
Resumo
Objetivo: descrever as concepções das enfermeiras acerca da assistência segura à mulher durante a gestação e parto. Método: trata-se de um estudo de abordagem qualitativa, do tipo descritiva, realizado em uma maternidade pública do município do Rio de Janeiro. Foram entrevistadas enfermeiras atuantes no atendimento pré-natal e na assistência ao parto normal. Utilizou-se a análise de conteúdo temática. Resultados: 11 enfermeiras participaram do estudo. A análise de conteúdo originou duas categorias temáticas, denominadas "Assistência obstétrica segura na concepção das enfermeiras" e "A promoção da segurança da assistência para as enfermeiras". As enfermeiras consideram que a segurança da assistência obstétrica é obtida por meio do uso de protocolos e normas técnicas preconizadas pelo Ministério da Saúde, buscando proporcionar uma assistência livre de erros e danos, sem intervenções desnecessárias e através de um cuidado humanizado, com profissionais capacitados e infraestrutura apropriada. A promoção da segurança ocorre quando há a prevenção de riscos, a valorização do protagonismo da mulher e o respeito à fisiologia do parto, através de uma assistência individualizada. Conclusão: nas concepções das enfermeiras, a assistência segura à mulher durante a gestação e parto, é quando quanto há um cuidado humanizado e individualizado, sem intervenções desnecessárias, através da adesão às recomendações técnicas, com profissionais capacitados e uma infraestrutura adequada, de forma a minimizar os erros e os danos assistenciais. As enfermeiras destacam a importância de estimular a autonomia da mulher, através de uma equipe acolhedora e capacitada, que forneça as informações e orientações necessárias contribuindo para a segurança da mulher.
\end{abstract}

Palavras-chave: Enfermagem obstétrica; Segurança do paciente; Qualidade da assistência à saúde.

\begin{abstract}
Objective: to describe nurses' conceptions about safe care for women during pregnancy and childbirth. Method: this is a qualitative, descriptive study, carried out in a public maternity hospital in the city of Rio de Janeiro. Nurses working in prenatal care and in assisting normal birth were interviewed. Thematic content analysis was used. Results: 11 nurses participated in the study. The content analysis gave rise to two thematic categories, called "Safe obstetric care in the nurses' conception" and "The promotion of safe care for nurses". Nurses consider that the security of obstetric care is obtained through the use of protocols and technical standards recommended by the Ministry of Health, seeking to provide assistance free of errors and damages, without unnecessary interventions and through humanized care, with trained and qualified professionals. appropriate infrastructure. The promotion of safety occurs when there is the prevention of risks, the valorization of the role of women and respect for the physiology of childbirth, through individualized assistance. Conclusion: in the nurses' conceptions, safe assistance to women during pregnancy and childbirth, is when there is a humanized and individualized care, without unnecessary interventions, through adherence to technical recommendations, with trained professionals and an adequate infrastructure, in order to minimize errors and damages. Nurses highlight the importance of encouraging women's autonomy, through a welcoming and trained team, which provides the necessary information and guidance, contributing to women's safety.
\end{abstract}


Keywords: Obstetric nursing; Patient safety; Quality of health care.

\section{Resumen}

Objetivo: describir las concepciones de las enfermeras sobre el cuidado seguro de la mujer durante el embarazo y el parto. Método: se trata de un estudio cualitativo, descriptivo, realizado en una maternidad pública de la ciudad de Río de Janeiro. Se entrevistó a enfermeras que trabajan en la atención prenatal y en la asistencia al parto normal. Se utilizó análisis de contenido temático. Resultados: participaron 11 enfermeras en el estudio. El análisis de contenido dio lugar a dos categorías temáticas, denominadas "Atención obstétrica segura en la concepción de las enfermeras" y "La promoción de la atención segura para las enfermeras". Las enfermeras consideran que la seguridad de la atención obstétrica se obtiene mediante el uso de protocolos y estándares técnicos recomendados por el Ministerio de Salud, buscando brindar una asistencia libre de errores y daños, sin intervenciones innecesarias y a través de una atención humanizada, con profesionales capacitados e infraestructura adecuada. La promoción de la seguridad se da cuando existe la prevención de riesgos, la valorización del rol de la mujer y el respeto a la fisiología del parto, a través de la atención individualizada. Conclusión: en las concepciones de las enfermeras, la asistencia segura a la mujer durante el embarazo y el parto, es cuando hay una atención humanizada e individualizada, sin intervenciones innecesarias, mediante la adherencia a las recomendaciones técnicas, con profesionales capacitados y una infraestructura adecuada, a fin de minimizar los errores. y daños a la atención. Las enfermeras destacan la importancia de fomentar la autonomía de las mujeres, a través de un equipo acogedor y capacitado, que brinda la información y orientación necesarias, contribuyendo a la seguridad de las mujeres.

Palabras clave: Enfermería obstétrica; Seguridad del paciente; Calidad de la atención de salud.

\section{Introdução}

A gestação é uma experiência marcante vivenciada por muitas mulheres e durante esse processo ocorrem diversos procedimentos que vão desde o pré-natal até o parto. Durante anos muitas mulheres morreram por falta de assistência adequada no ciclo gravídico-puerperal, mas com o surgimento de políticas públicas voltadas para a saúde da mulher tem-se contribuído para a redução da mortalidade materna, através de uma assistência humanizada, segura e de qualidade nas instituições de saúde.

Em 2013, a Organização Mundial de Saúde estimou a mortalidade materna em cerca de 289.000 mulheres, representando uma taxa de mortalidade global de 210 mortes maternas para 100.000 nascidos vivos (Carvalho; Göttems \& Pires, 2015). No Brasil, foram registrados 38.919 óbitos maternos no período de 1996 a 2018. No ano de 2018, a Razão de Mortalidade Materna (RMM) foi de 59,1 óbitos maternos por 100 mil nascidos vivos (Brasil, 2020). A persistência desta RMM elevada está relacionada com as caraterísticas predominantes da assistência obstétrica brasileira, como a intensa medicalização, o uso de intervenções desnecessárias e iatrogênicas, a prática abusiva da cesariana, a ausência de privacidade da mulher e desrespeito a sua autonomia, entre outros, são fatores influentes nos riscos de mortalidade materna e perinatal (Brasil, 2011).

Para reverter este cenário, as políticas governamentais estimulam a qualificação e atuação de enfermeiras obstétricas na assistência ao parto normal para favorecer a redução destes indicadores de mortalidade e implementação da humanização da assistência ao pré-natal, parto e nascimento por meio do respeito à dignidade e aos direitos femininos, assim como à fisiologia do corpo da mulher na gestação e parto (Gomes et al, 2014).

Além desses incentivos, outras medidas foram adotadas para a melhoria da qualidade e segurança da assistência prestada no Sistema Único de Saúde (SUS), como a criação do Programa Nacional de Segurança do Paciente (PNSP) em 2013 e, no ano seguinte, a publicação do documento de referência para a segurança e qualidade nos serviços de atenção materna e neonatal (Minuzzi, Salum, Locks, Amante \& Matos, 2016; Brasil, 2014).

A segurança do paciente abrange um conjunto de ações destinadas à proteção dos pacientes contra as situações de riscos, incidentes e danos que podem ocorrer durante o processo de assistência. De acordo com o PNSP, os eventos com ou sem dano são denominados de incidentes e envolvem circunstâncias notificáveis, podendo ser erros na execução da assistência, falhas na comunicação, inadequação de pessoal e deficiências na manutenção de equipamentos por exemplo. Os incidentes com dano físico, psicológico ou social são denominados de eventos adversos (Brasil, 2014). 
É recomendado também o envolvimento dos gestores, profissionais de saúde e usuários dos serviços de saúde para a modificação dos fatores políticos, culturais, educacionais, organizacionais e profissionais que podem interferir na qualidade e segurança da atenção em saúde (Minuzzi et al., 2016; Brasil, 2014).

Diante do exposto, presume-se que uma assistência segura na atenção obstétrica envolve a oferta de um cuidado humanizado, baseado na fisiologia do gestar e parir, que valoriza a autonomia feminina e tenha conformidade com as recomendações ministeriais. A partir de tais premissas, torna-se necessário o conhecimento dos sentidos e significados que as enfermeiras atribuem à assistência obstétrica segura.

Devido à necessidade de compreender os pontos de vista destas profissionais, este estudo teve como questionamento: Quais as concepções das enfermeiras sobre a assistência obstétrica segura à mulher no período de gestação e parto?

O objetivo deste estudo foi descrever as concepções das enfermeiras acerca da assistência segura à mulher durante a gestação e parto.

O estudo se justifica pela relevância do tema da segurança e qualidade da assistência na atenção obstétrica em face dos indicadores morbimortalidade materna e das inadequações dos cuidados oferecidos às mulheres grávidas observadas nos serviços de saúde do país, o que ressalta a importância da adesão às recomendações técnicas de humanização do parto e nascimento.

\section{Metodologia}

Trata-se de pesquisa com abordagem qualitativa, do tipo descritiva, conduzida em uma maternidade pública do município do Rio de Janeiro. Essa instituição dispõe de ambulatório de pré-natal, complementar à rede básica da região adstrita para a assistência pré-natal, e centro obstétrico, onde há o atendimento das gestantes classificadas de baixo risco e de risco obstétrico no momento do parto e nascimento.

Os critérios de inclusão foram enfermeiras (os) que prestam assistência às mulheres no período da gestação e parto, e trabalham nos setores de pré-natal e centro obstétrico. Os critérios de exclusão são profissionais que atuam a menos de quatro meses nesses cuidados obstétricas.

A coleta de dados aconteceu nos meses de maio e junho do ano 2017 e se deu através de entrevistas individuais com apoio de roteiro semiestruturado, sendo esse constituído por duas partes. A primeira parte havia questões que versavam sobre a caracterização profissional das enfermeiras e, a segunda, constituída pelo roteiro de perguntas propriamente dito, acerca do entendimento da depoente sobre assistência obstétrica segura e como se promove a segurança desta assistência. As entrevistas tiveram variação quanto à sua duração, de 06:25 minutos a 22:42 minutos.

Foram selecionados 13 enfermeiros para entrevista de acordo com os setores de atuação, mas dois foram excluídos por atuarem menos de quatro meses na consulta de enfermagem pré-natal e assistência ao parto normal, totalizando 11 participantes. No ambulatório, trabalham apenas quatro enfermeiras. Entre essas profissionais, houve dificuldade de captação de duas delas para o agendamento das entrevistas e a exclusão de uma enfermeira ocorreu pelo tempo de trabalho no setor ser inferir ao previsto nos critérios de seleção da pesquisa, sendo entrevistada apenas uma enfermeira deste setor. Houve também sucessivas tentativas de agendamento com duas profissionais para entrevista no centro obstétrico, mas que não resultaram em sucesso, perfazendo o total de dez participantes atuantes neste setor da maternidade. Apenas um é do sexo masculino. Em virtude disso, todos os participantes são aqui designados pelo gênero feminino.

Os depoimentos foram analisados por meio da técnica da análise de conteúdo temática, que prevê três etapas. A primeira é a pré-análise dos depoimentos, quando há transcrição e organização das entrevistas, assim como a leitura flutuante das mesmas. Em seguida, houve a exploração do material, sendo estabelecida a codificação por equivalência semântica dos códigos segundo a similaridade dos sentidos e significados presentes nas unidades de registro (UR), e construção das 
categorias temáticas. Por fim, o tratamento dos resultados, realizando-se a inferência e a interpretação segundo a literatura científica e pressupostos da qualidade e segurança da assistência na área obstétrica.

A análise de conteúdo possibilita o acesso aos múltiplos conteúdos, explícitos ou não, de um texto, permitindo a análise das representações ou concepções sobre algum objeto ou tema, assim como do repertório semântico ou sintático de um certo grupo social ou profissional. Essa técnica de análise tem como objeto a palavra, isto é, o aspecto individual e atual da linguagem, focalizando nos significados atribuídos pelos sujeitos acerca de um determinado fenômeno da realidade, com intento de conhecer o que está por trás das palavras sobre as quais se debruça o pesquisador (Bardin, 2011).

O direcionamento da análise se deu a partir do esquema interrogativo com as seguintes questões: $\mathrm{O}$ que é assistência obstétrica segura? Como se deve realizá-la? Isto permitiu agrupar as unidades de significação a partir destas questões conforme o sentido apresentado no texto e estabelecer as codificações segundo a agregação dos sentidos e significados semelhantes. Essa fase de exploração ocorreu no Programa Microsoft Office Excel® 2007, que facilitou o agrupamento dos códigos encontrados, o estabelecimento das categorias temáticas e a contagem de suas UR correspondentes.

O presente estudo atendeu os princípios éticos previstos na legislação pertinente à pesquisa com seres humanos, sendo aprovado pelo Comitê de Ética e Pesquisa da Secretaria Municipal de Saúde do Rio de Janeiro (SMSRJ), com parecer $n^{\circ}$ 1.956.925. Todos os participantes assinaram o Termo de Consentimento Livre e Esclarecido.

\section{Resultados}

A maioria das enfermeiras entrevistadas atuavam no setor do centro obstétrico e apenas uma trabalhava no ambulatório de pré-natal. O tempo médio de atuação na profissão foi de 10,4 anos, variando de 1 a 28 anos. Quanto à qualificação profissional, seis profissionais são especialistas em enfermagem obstétrica, três enfermeiras são especializadas em terapia intensiva, enfermagem do trabalho e saúde da mulher. Duas enfermeiras concluíram o curso de mestrado acadêmico.

A análise de conteúdo temática originou duas categorias temáticas ou temas, sendo a primeira "Assistência obstétrica segura na concepção das enfermeiras", abarcando 398 UR, e a segunda categoria "A promoção da segurança da assistência para as enfermeiras", abrangendo 185 UR.

\subsection{Assistência obstétrica segura na concepção das enfermeiras}

As enfermeiras consideram que a segurança da assistência obstétrica é obtida por meio do uso de protocolos e normas técnicas preconizadas pelo Ministério da Saúde, buscando proporcionar uma assistência livre de erros, danos e iatrogenias à clientela, sendo necessário respeitar a fisiologia da gestação e do parto, proporcionar assistência sem intervenções desnecessárias, além de realizar escuta qualificada e acolhimento à mulher, contemplando a humanização da assistência à mulher, como o respeito, a privacidade e o conforto.

Um parto seguro é aquele em que eu busco aproximar ao máximo as minhas práticas daquilo que é preconizado pelo Ministério da Saúde, na questão das boas práticas, e conduzir no caso de encontrar com alguma intercorrência, conduzir de acordo com os protocolos em si, e estar sempre avaliando essa mulher de maneira correta, seguindo os protocolos. (E-2).

É você se cercar de todos os cuidados e todas as normas, cumprir todas as normas preconizadas para você conseguir uma assistência adequada, uma identificação correta do bebê e ter os materiais para você atender a mulher com segurança. (E-4). 
Entendo como segurança dessa mulher, como assegurar a ela pelo menos o respeito, o acolhimento, a humanização que todo mundo defende, é buscar minimamente o respeito e assegurar a ela tudo que a gente busca como qualidade, (...) assegurar saúde, exames e escuta mais qualificada, no sentido de você oferecer exatamente a acessibilidade que tanto se fala no SUS. (E-12).

A assistência obstétrica segura para as participantes é também aquela realizada por profissional com conhecimento atualizado, competência técnica adequada e que dispõe de infraestrutura apropriada nas unidades de saúde, como o ambiente e os recursos humanos e materiais necessários para a segurança do cuidado, como sugerem os seguintes depoimentos:

Primariamente acho que a segurança obstétrica se dá pela habilidade e competência que se desenvolve ao longo da sua formação. Acho que o primeiro ponto chave de segurança seria você ter destreza e competência. (E-11).

(...) E que haja profissionais qualificados dentro da equipe, não necessariamente precisa ser um obstetra ou mais de um obstetra, mas que se tenham pessoas que saibam manejar essa parturiente. (E-8).

Eu acho que os profissionais são difíceis, os profissionais precisam se atualizar. Eu não estou falando só da enfermagem, mas a enfermagem também. Eu acho que a medicina e a instituição precisam muito se atualizar. (E -9).

Aqui na unidade, a estrutura da sala de parto dificulta muito a assistência, por exemplo, a mulher precisa deambular e ela só tem esse espaço, nós só temos um banheiro, ela tem que passar por todos os boxes, e não deveria ser assim. $(E-7)$.

\subsection{A promoção da segurança da assistência para as enfermeiras}

A promoção da segurança para as participantes ocorre quando há a prevenção de riscos, valorização do protagonismo da mulher e respeito à fisiologia do parto. Para tal, a assistência deve estar pautada na execução de cuidados humanizados, conforme as recomendações, e na vigilância do bem-estar materno e do bebê para garantir a segurança da assistência prestada.

É você auscultar o bebê de tempo em tempo e de forma correta, avaliar a mulher toda vez que for necessário, acompanhá-la de perto e não a deixar abandonada, nem ela e nem o bebê, (...) é você acompanhar a mulher, ajudar a tirar as dúvidas durante o pré-natal, deixar a mulher empoderada para ela saber o que acontecerá e sobre o que é um trabalho de parto (...). (E-5).

É evitar ao máximo de intervenções para essa mulher e saber aplicar as tecnologias não invasivas. E aplicar as intervenções no momento certo e adequado e de forma que não prejudique nem ela e nem o bebê. (E-9).

As profissionais também destacaram os cuidados respeitosos à autonomia da mulher e à fisiologia do parto, não ser invasivo, atuar com acolhimento, orientação e assistência individualizada. Elas mencionaram a importância do acesso às informações durante o pré-natal, do respeito aos direitos e do incentivo ao protagonismo da mulher, com o uso de práticas não invasivas no parto para a promoção da assistência segura e humanizada para a mulher e seu filho.

(...) no pré-natal, você pode trabalhar a mulher, informar sobre o trabalho de parto, o que acontece no parto e sua evolução. Em uma mulher que foi informada, esclarecida e bem orientada durante o pré-natal, você consegue trabalhar muita coisa para oferecer uma assistência melhor durante o parto, em termos de segurança e qualidade, $e$ em termos de humanização. (E-6). 
Então, eu acho que é bem importante, ela [gestante] se sentir segura, saber quem está acolhendo, saber todas as possibilidades terapêuticas, não farmacológicas ou farmacológicas, seja lá quem for aplicá-las, fazer os procedimentos, mas que ela tenha essa segurança também, isso favorece com certeza o nascimento seguro e tranquilo para ela e para o bebê. (E-11).

\section{Discussão}

Desenvolver uma assistência de enfermagem de maneira adequada é fundamental para promover um cuidado seguro à mulher. Rocha e Andrade (2017) apontaram que o ato de proporcionar uma assistência humanizada e de qualidade permite à mulher gestante se sentir acolhida e bem assistida nos serviços de saúde.

A enfermagem obstétrica tem um papel importante quando o assunto é qualidade na assistência, pois possui uma função considerável no processo de gestação ao nascimento. Mas, apesar de vários alcances, há desafios a serem alcançados, sendo necessário que a enfermeira obstetra assuma o seu lugar de gestora do cuidado obstétrico e neonatal para que a melhoria do cuidado se efetive na prática de fato (Backes et al., 2017).

A pesquisa evidenciou que a assistência obstétrica segura é aquela que se caracteriza por um cuidado pautado nos protocolos e normas técnicas e seja exercido com respeito, habilidade e competência profissional durante o pré-natal e parto. A assistência obstétrica deve ser baseada nas evidências científicas para melhoria dos cuidados à mulher e ao bebê (Santos \& Araújo, 2016).

A implantação das boas práticas de assistência conforme as recomendações e normas técnicas, relatada pelas enfermeiras, favorece o cuidado humanizado preconizado nas políticas de saúde. A assistência de enfermagem obstétrica tem promovido impacto positivo na qualidade da assistência das mulheres e seus bebês pela perspectiva de cuidado integral e humanizado destas profissionais (Peripolli et al., 2019; Sousa et al., 2016).

O cuidado de enfermagem na atenção obstétrica tem contribuído para melhorias da assistência por meio da formação de vínculo, da escuta qualificada e do acolhimento durante o pré-natal e parto, assim como do uso dos métodos não farmacológicos para alívio da dor, estímulo do contato pele a pele mãe-bebê e utilização restrita de intervenções desnecessárias, como a episiotomia, ocitocina e amniotomia (De Bortoli, Prates, Perez, Champe, Wilhelm, \& Ressel, 2020; Vieira, Santos, Silva, \& Sanches, 2016).

A segurança do paciente está diretamente associada à qualidade em saúde e vice-versa, sendo vista como um importante componente da qualidade e compreendida como a prevenção, a melhoria e a correção dos resultados adversos ou dos danos decorrentes do processo assistencial, que envolve tanto os pacientes quanto os profissionais de saúde (Marcolin, 2015).

A mudança no modo como a assistência vem sendo prestada é considerada de capital relevância para o alcance da qualidade e segurança da assistência materna e neonatal. Nessa direção, o cuidado materno respeitoso integra as recomendações da OMS para a atenção pré-natal e ao parto, pois se refere aos cuidados às mulheres norteados pelos princípios da dignidade, privacidade e confidencialidade, a fim de garantir a integridade física, permitir que elas tomem decisões informadas e recebam apoio necessário e tratamento adequado (World Health Organization, 2018).

Nessa direção, as participantes do presente estudo concebem a assistência obstétrica segura à mulher e ao bebê como aquela na qual os profissionais dispõem de conhecimentos técnicos atualizados e estão adequadamente capacitados. Azevedo et al. (2020) também trazem essa necessidade de atualização constante de conhecimento das profissionais de enfermagem que exercem sua atividade em maternidade. Dentre os papéis da enfermagem, um deles é de se responsabilizar pelo aperfeiçoamento da equipe de enfermagem, sendo ressaltado pelas enfermeiras entrevistadas a importância da atualização e qualificação profissional para garantir uma assistência obstétrica segura. Esse ponto de vista é corroborado por Dias, Mekaro, 
Tibes e Zem-Mascarenhas (2014), ao considerarem que a educação continuada e a capacitação de enfermeiros e enfermeiras são essenciais na promoção da segurança do paciente.

Além da capacitação profissional, a infraestrutura adequada do serviço também foi considerada como essencial para a segurança da assistência pelas enfermeiras. A infraestrutura adequada destacou-se no estudo de Sabino, Costa, Bracarense, Duarte e Simões (2017), uma vez que o cuidado deve ser constituído por boas práticas e adequação tecnológica e da infraestrutura para viabilizar uma assistência eficaz e de qualidade, que contemple o pessoal e os recursos materiais necessários.

É importante ressaltar que, para a promoção da segurança na assistência à gestante, deve-se atentar também para os cuidados de segurança do paciente, tais como: higienização das mãos, identificação correta da mãe e do bebê, reconhecimento oportuno das complicações obstétricas, monitoramento e prevenção dos eventos adversos, administração segura de medicamentos, prevenção de quedas, bem como estimular a participação ativa da mulher na assistência a ela prestada (Brasil, 2014).

As enfermeiras destacam a utilização de tecnologias não invasivas ao corpo da mulher para a promoção da segurança. A enfermagem obstétrica ressalta o uso das tecnologias de cuidado preconizados pelo Ministério da Saúde, sendo essas um meio capital para assegurar o respeito da fisiologia da gestação e parto, sem intervenções desnecessárias, e a garantia de um cuidado humanizado, além de destacar a centralidade do cuidado na mulher e a relevância das práticas de educação em saúde no processo assistencial.

Para tal, os profissionais de saúde precisam estar capacitados e se perceberem responsáveis pelo atendimento humanizado e seguro às mulheres, aos recém-nascidos, aos acompanhantes e aos familiares. A humanização inclui a valorização da dimensão subjetiva e social nas práticas de atenção, com ênfase no respeito ao gênero, raça, etnia, orientação sexual e populações específicas (Brasil, 2014).

O movimento da humanização é baseado em uma assistência acolhedora e respeitosa à parturiente, e, além disso, propôs que sejam adotadas as melhores evidências científicas, representando uma mudança paradigmática no modelo obstétrico dominante no país (Sousa et al., 2016). Essa mudança foi observada em uma pesquisa sobre as tecnologias não invasivas de cuidado na perspectiva da desmedicalização, no qual as enfermeiras desenvolvem um cuidado pautado na relação humana, na integralidade e no protagonismo feminino, contribuindo para a humanização da assistência preconizada nos programas de saúde brasileiros (Prata et al., 2019).

O sistema de saúde avançou e incluiu enfermeiras obstetras para contribuírem com esta mudança na atenção obstétrica, de uma assistência intervencionista e invasiva ao corpo da mulher para um cuidado qualificado, seguro, respeitoso e efetivo, ao corroborar com os princípios da humanização e da integralidade previstos no SUS e, assim, favorecer a melhoria da qualidade assistencial (Castro, Narchi, Lopes, Macedo e Souza et al., 2017). Tais avanços refletem que a enfermagem na atenção obstétrica tem o potencial de participar ativamente na promoção da segurança da assistência, visto que sua formação profissional dá ênfase nas ações de educação em saúde e participação ativa do usuário nos cuidados, como destacaram as enfermeiras entrevistadas.

As enfermeiras obstétricas são profissionais adequadas para o acompanhamento do trabalho de parto e partos normais em mulheres saudáveis, por apresentarem características profissionais que envolvem a menor utilização de intervenções em seus cuidados, o favorecimento da segurança e respeito ao processo fisiológico do parto e a promoção da dignidade e autonomia da mulher, que corroboram com as mudanças nas práticas e rotinas institucionais preconizadas para a humanização no parto e nascimento (Vargens, Silva \& Progianti, 2017).

O cuidado humanizado também foi reconhecido pela enfermagem como necessário para alcançar a segurança da assistência obstétrica, sendo essa identificada pelas enfermeiras participantes do estudo como aquela que se efetiva por meio 
do cuidado não invasivo, no qual a assistência é integral e há a utilização de técnicas não invasivas durante o processo de parto, com respeito à sua fisiologia e emprego de intervenções quando são de fato estritamente necessários. Portanto, no âmbito da equipe multiprofissional, a enfermeira obstétrica é considerada menos aderente às intervenções, que usa os métodos não farmacológicos para o alívio da dor, dá suporte emocional, busca favorecer o conforto para as mulheres e propiciar uma vivência menos traumática e mais segura do parto (Vogt et al., 2014).

Outro aspecto abordado pelas profissionais entrevistadas foi a monitorização contínua da mulher e do bebê como meio de viabilizar a segurança nos cuidados. Essa avaliação contínua também foi destacada por Marcolin (2015), ao ressaltar sua importância no cuidado do paciente prestado pelos serviços por cada membro da equipe, assim como no ambiente assistencial dos serviços de saúde, em relação à segurança dos equipamentos e recursos no local onde o cuidado é operacionalizado.

Outro aspecto importante para a segurança da assistência obstétrica é a adesão aos protocolos ministeriais. As enfermeiras consideram que a postura de vigilância do bem-estar materno e do bebê conforme recomenda os protocolos e normas técnicas é indispensável na promoção da segurança da assistência. O uso de protocolos assistenciais é uma ferramenta importante para a realização de boas práticas com ênfase na humanização da assistência, a fim de prevenir divergências no processo de cuidar, direcionar a prática e reduzir fragilidades para o cuidado da mulher. Além disso, um protocolo de boas práticas como o cuidado integral à mãe e ao recém-nascido garante segurança e conforto para ambos (Piler, Wall, Aldrighi, Benedet, Silva \& Szpin, 2019).

Para Marcolin (2015) a promoção da segurança obstétrica requer uma assistência pautada na prevenção de riscos e minimização os danos, visto que é o mais importante componente da qualidade e pode ser definida como a prevenção, a melhoria e a correção dos resultados adversos provenientes do processo assistencial, tanto no âmbito dos pacientes que recebem os cuidados quanto em relação aos profissionais que os realizam. Nessa perspectiva, recomendam-se diretrizes clínicas baseadas em evidências; listas de verificação padronizadas, processos de comunicação adequados e trabalho em equipe, com comprometimento e responsabilidade pela continuidade e oferta dos cuidados ao paciente (Rodrigues, Pereira, Pessanha \& Penna, 2021).

As enfermeiras também afirmam que a segurança é alcançada por meio da humanização da assistência às mulheres com referência ao acesso à informação e ao protagonismo feminino. A assistência obstétrica centrada nas necessidades da parturiente requer que o profissional garanta o direito à autonomia da mulher, o acesso às informações de qualidade e a participação ativa da mesma no trabalho de parto e parto, almejando o protagonismo feminino a fim de que ela possa ter a consciência de sua capacidade de exercer escolhas e de parir a partir da visão do parto como um processo natural e fisiológico (Silva, Nascimento \& Coelho, 2015).

Corroborando com esta perspectiva, as mulheres devem ser estimuladas a atuar como sujeito do acontecimento, pois elas têm o direito de participar das decisões sobre o nascimento. Destaca-se, entretanto, que as mesmas ainda têm dificuldades em assumir um papel ativo no trabalho de parto devido a cultura de submissão a que estão submetidas. $\mathrm{O}$ desrespeito e os maus-tratos durante o processo assistencial podem causar danos emocionais e físicos no parto, sendo considerados como eventos adversos para a mulher e o bebê (Rodrigues et al., 2021).

As enfermeiras participantes do estudo consideram que a segurança da assistência abarca a utilização dos métodos não farmacológicos e não invasivos de alívio à dor a fim de favorecer a progressão fisiológica do trabalho de parto. O alívio da dor durante o trabalho de parto é um tema amplamente difundido e há uma grande variedade de cuidados para promoção do conforto e relaxamento da parturiente, tais métodos promovem uma relação de apoio e segurança no momento do parto (Guida et al., 2017). Silva et al. (2015) dizem que é importante considerar o direito à autonomia, o acesso às informações e a participação da mulher no trabalho de parto, para que ela se perceba como protagonista do seu próprio parto e tenha 
consciência desse evento como um fenômeno fisiológico e natural da vida reprodutiva feminina.

$\mathrm{O}$ acolhimento e a orientação também se revelaram neste estudo como necessários ao cuidado individualizado e seguro. Ao acolher e fornecer as orientações necessárias às mulheres grávidas, as enfermeiras têm a possibilidade de promover a interação e a participação das gestantes no processo assistencial, permitindo-as manifestar seus anseios e questionamentos, possibilitando a troca de conhecimento, a comunicação, o diálogo e a interação entre a gestante e o profissional. As orientações individualizadas, conforme a necessidade de cada mulher, são primordiais para efetivar a qualidade e segurança durante a gestação, parto e nascimento (De Bortoli et al., 2020).

\section{Limitações do estudo}

O tema segurança da assistência obstétrica ainda é recente no meio acadêmico e institucional, demonstrando a necessidade de se avançar o conhecimento sobre essa temática e abarcar outras metodologias e cenários de pesquisa. $\mathrm{O}$ fato desta pesquisa ter sido realizada somente em uma instituição de saúde não permite generalizar os aqui resultados descritos, pois foram advindos das perspectivas de um grupo particular de enfermeiras, sendo indicado ampliar estudos que abarquem a diversidade de cenários e atores da atenção obstétrica, sobretudo as mulheres.

\section{Contribuições para a área}

Este estudo poderá contribuir com os profissionais de enfermagem, da equipe multiprofissional e dos gerentes dos serviços de saúde da área obstétrica para estimulá-los sobre a importância do tema da segurança e qualidade da assistência no pré-natal e parto, a fim de buscar estratégias de adesão de todos aos princípios da humanização da assistência, às recomendações ministeriais e às normas técnicas, assim como convidá-los a estabelecer os esforços necessários para oferecer uma assistência qualificada, livre de riscos e sem iatrogenias, de modo a efetivar melhorias nos indicadores de morbimortalidade materna e perinatal.

Além disso, o estudo poderá suscitar reflexões sobre a segurança da assistência e a responsabilidade dos profissionais e gestores na garantia da qualidade da atenção à clientela, para que possam direcionar melhor suas decisões e comportamentos em prol de um cuidado seguro e baseado nas evidências científicas.

\section{Considerações Finais}

O presente estudo permitiu conhecer as concepções das enfermeiras acerca da assistência obstétrica segura. Elas consideram que a assistência segura ocorre quando há humanização no cuidado, adesão às recomendações técnicas, profissionais capacitados e infraestrutura adequada e necessária para minimizar erros e danos decorrentes da assistência.

Para as enfermeiras, o modelo de assistência obstétrica deve ser sem intervenções desnecessárias para que o parto ocorra de modo mais natural possível e sem iatrogenias. Para tal, essas profissionais propõem uma abordagem integral, humanizada e respeitosa à mulher, conforme preconizam os programas ministeriais voltados para humanização da assistência. Além dessas concepções, elas mencionaram a importância da competência e atualização do profissional para proporcionar um cuidado livre de riscos e danos, e da adequação do conhecimento profissional e das iniciativas de capacitação em serviço para que seus cuidados sejam norteados pelas evidências científicas. As enfermeiras consideram que as intervenções devem realizadas sob estrita indicação e de forma correta, evitando-se assim complicações e danos para as mulheres e seus bebês.

As entrevistadas também destacaram a importância de se estimular a autonomia da mulher para que a mesma consiga exercer o seu direito de tomar decisões acerca do seu cuidado durante a gestação e parto. Para tal, as enfermeiras consideram essencial que a equipe apresente atitude acolhedora e forneça informações e orientações necessárias para que a gestante ou parturiente possa exercer escolhas conscientes, contribuindo para a segurança deste cuidado. 
Essas concepções denotam necessidade de se ampliar esforços para a mudança do modelo obstétrico dominante para a consolidação da humanização da assistência e, sobretudo, avançar nos conhecimentos acerca do PNSP, uma vez que as falas das enfermeiras contemplaram de forma genérica a temática do segurança das pacientes na atenção pré-natal e parto. Apesar de terem citado a identificação correta e a prevenção de quedas, as entrevistadas não especificaram demais metas e propostas programáticas da segurança do paciente.

Diante das concepções das enfermeiras aqui descritas, verifica-se que a segurança da assistência obstétrica na assistência pré-natal e ao parto ainda tem um grande caminho a percorrer, indicando-se a divulgação dos pressupostos dos programas de segurança do paciente e o fortalecimento dos princípios do programa de humanização por meio de estratégias de educação permanente das equipes desta aérea assistencial, uma vez que ambos os programas estão direcionados para efetivar uma maternidade segura e melhorias dos indicadores de saúde materna e neonatal.

Por fim, é importante ressaltar que pesquisas devem ser realizadas acerca da temática da segurança da assistência na área obstétrica, com vistas a contemplar outras perspectivas, cenários e desenhos metodológicos capazes de mensurar os indicadores de segurança e avaliar os processos de implantação das recomendações dos programas de segurança e humanização da assistência às mulheres no ciclo gravídico-puerperal.

\section{Referências}

Azevedo, L. S., Costa, S., Donaduzzi, D. S. S., Fettermann, F. A., Bordignon, J., \& Rosa, A. B. (2020). Conhecimento de enfermeiras obstétricas em relação aos métodos não farmacológicos para o alívio da dor no trabalho de parto. Revista Vivências, 16(30), 115-125. http://webcache.googleusercontent.com/search?q=cache:L7OgPV4g-o4J:revistas.uri.br/index.php/vivencias/article/view/118/93+\&cd=1\&hl=pt$\mathrm{BR} \& \mathrm{ct}=\mathrm{clnk} \& \mathrm{gl}=\mathrm{br}$

Backes, M. T. S., Ribeiro, L. N., Amorin, T. S., Vieira, B. C., Souza, J., Dias, H. H. Z. R. \& Zampieri, M. F. M. (2017). Desafios da gestão do cuidado de enfermagem para a qualidade da atenção obstétrica e neonatal em maternidades públicas brasileiras. Investigação Qualitativa em Saúde. 2, 411-420. http://proceedings.ciaiq.org/index.php/ciaiq2017/article/view/1232/1193

Bardin, L. (2011). Análise de Conteúdo. Edições 70.

Brasil. (2014). Agência Nacional de Vigilância Sanitária. Serviços de atenção materna e neonatal: segurança e qualidade. Brasília: ANVISA, 103 (Tecnologia em serviços de saúde). https://www20.anvisa.gov.br/segurancadopaciente/index.php/publicacoes/item/servicos-de-atencao-materna-e-neonatalseguranca-e-qualidade

Brasil. (2020). Ministério da Saúde. Secretaria de Atenção Primária à Saúde. Brasil reduziu 8,4\% a razão de mortalidade materna e investe em ações com foco na saúde da mulher. https://aps.saude.gov.br/noticia/8736\#: :text=O\%20Brasil\%20conseguiu\%20reduzir\%20em,anterior\%20era\%20de\%2064\%2C5.

Brasil. (2011). Ministério da Saúde. Política Nacional de Atenção Integral à Saúde da Mulher: Princípios e Diretrizes. Editora do Ministério da Saúde. http://bvsms.saude.gov.br/bvs/publicacoes/politica_nacional_mulher_principios_diretrizes.pdf

Carvalho, E. M. P., Göttems, L. B. D. \& Pires, M. R. G. M. (2015). Adesão às boas práticas na atenção ao parto normal: construção e validação de instrumento. Revista Escola de Enfermagem da USP, 49(6), 890-898. http://www.revistas.usp.br/reeusp/article/view/108367

Castro, C. M., Narchi NZ, Lopes G. A., Macedo C. M., \& Souza A. C. (2017). Entre o ideal e o possível: experiências iniciais das obstetrizes no Sistema Único de Saúde de São Paulo. Saúde Soc. 26(1), 312-323. http://www.scielo.br/pdf/sausoc/v26n1/1984-0470-sausoc-26-01-00312.pdf

De Bortoli, C. de F. C., Prates, L. A., Perez, R. de V., Champe, T. da S., Wilhelm, L. A., \& Ressel, L. B. (2020). A consulta de enfermagem: contribuições na atenção pré-natal. Research, Society and Development, 9(8), e458985236. http://doi.org/10.33448/rsd-v9i8.5236

Dias, J. D., Mekaro, K. S., Tibes, C. M. S., \& Zem-Mascarenhas, S. H. (2014). Compreensão de enfermeiros sobre segurança do paciente e erros de medicação. Revista Mineira de enfermagem, 18(4), 866-873. http:// http://www.reme.org.br/artigo/detalhes/969

Gomes, A. R. M, Pontes, D. S., \& Pereira, C. C. A., Brasil, A. O. M., \& Moraes, L. C. A. (2014). Assistência de enfermagem obstétrica na humanização do parto normal. Revista Recien, 4(11), 23-27. http://www.recien.com.br/index.php/Recien/article/view/73/137

Guida, N. F. B., Pereira, A. L. F., Lima, G. P. V., Zveiter, M., Araújo, C. L. F., \& Moura, M. A. V. (2017). Conformidade das práticas assistenciais de enfermagem com as recomendações técnicas para o parto normal. Revista Rene, 18(4), 543-550. http://www.periodicos.ufc.br/rene/article/view/20286/30818

Marcolin, A. C. (2015). Qualidade e segurança: caminhos para o sucesso do redesenho do modelo de cuidado obstétrico. Revista Brasileira de Ginecologia e Obstetrícia, 37(10), 441- 445. http://www.scielo.br/pdf/rbgo/v37n10/0100-7203-rbgo-37-10-00441.pdf

Minuzzi, A. P., Salum, N. C., Locks, M. O. H., Amante, L. N., \& Matos, E. (2016). Contribuições da equipe de saúde visando à promoção da segurança do paciente no cuidado intensivo. Escola Anna Nery, 20(1),121-129. http://dx.doi.org/10.5935/1414-8145.20160017

Peripolli, L. O., Rabelo, M.; Souza, S. R. R. K., Wall, M. L., Trigueiro, T. H., \& Godarth, K.M. (2019). Assistência ao parto sob a ótica de enfermeiras obstétricas. Revista Família, Ciclos de Vida e Saúde no Contexto Social REFACS, v.7, n.3, p.275-283, 2019. http://doi.org/10.18554/refacs.v7i3.3749 
Research, Society and Development, v. 10, n. 3, e37910313360, 2021

(CC BY 4.0) | ISSN 2525-3409 | DOI: http://dx.doi.org/10.33448/rsd-v10i3.13360

Piler, A. A., Wall, M. L., Aldrighi, J. D., Benedet, D. C. F., Silva, L. R. \& Szpin, C. C. (2019). Protocolo de boas práticas obstétricas para os cuidados de enfermagem no processo de parturição. Revista Mineira de Enfermagem, 23:e-1254, 1-8. http://doi.org/10.5935/1415-2762.20190102

Prata, J. A., Ares, L. P. M., Vargens, O. M. C., Reis, C. S. C., Pereira, A. L. F., \& Progianti, J. M. (2019). Tecnologias não invasivas de cuidado: contribuições das enfermeiras para a desmedicalização do cuidado na maternidade de alto risco. Escola Anna Nery, 23(2):e20180259, 1-8. http://doi.org/10.1590/2177-9465EAN-2018-0259

Rocha, A. C. \& Andrade, G. S. (2017). Atenção da equipe de enfermagem durante o pré-natal: percepção das gestantes atendidas na rede básica de Itapuranga - GO em diferentes contextos sociais. Revista Enfermagem Contemporânea, 6(1), 30-41. http://dx.doi.org/10.17267/2317-3378rec.v6i1.1153

Rodrigues, G. T., Pereira, A. L. F., Pessanha, P. S. A., \& Penna, L. H. G. (2021). Incidentes na assistência das parturientes e recém-nascidos: perspectivas das enfermeiras e médicos. Escola Anna Nery, .25 (2), e20200075. http://doi.org/10.1590/2177-9465-ean-2020-0075.

Sabino, V. G. R. S., Costa, N. S., Bracarense, C. R., Duarte, J. M. G., \& Simões, A. L. A. (2017). A percepção das puérperas sobre a assistência recebida durante o parto. Revista de Enfermagem da UFPE On-line. 11(10), 3913-9. http://doi.org/10.5205/reuol.12834-30982-1-SM.1110201728

Santos, H. F. L., \& Araújo, M. M. (2016). Políticas de Humanização ao pré-natal e parto: uma revisão de literatura. Revista Científica FacMais, 4(2), 54-64. http://revistacientifica.facmais.com.br/wp-content/uploads/2016/07/Artigo-6-POL\%C3\%8DTICAS-DE-HUMANIZA\%C3\%87\%C3\%83O-AO-PR\%C3\%89NATAL-E-PARTO.pdf.

Silva, A. L. S., Nascimento, E. R., \& Coelho, E. A. C. (2015). Práticas de enfermeiras para promoção da dignificação, participação e autonomia de mulheres no parto normal. Escola Anna Nery, 19(3), 424-431. http://doi.org/10.5935/1414-8145.20150056

Sousa, A. M. M, Souza, K. V., Rezende, E. M., Martins, E. F., Campos, D., \& Lansky, S. (2016). Práticas na assistência ao parto em maternidades com inserção de enfermeiras obstétricas, em Belo Horizonte, Minas Gerais. Escola Anna Nery, 20(2), 324-331. http://doi.org/10.5935/1414-8145.20160044

Vargens, O. M. C., Silva, A. C. V., \& Progiant, J. M. (2017). Contribuição de enfermeiras obstétricas para consolidação do parto humanizado em maternidades no Rio de Janeiro-Brasil. Esc Anna Nery, 21(1), 1-8. http://doi.org/10.5935/1414-8145.20170015

Vieira, M. J O., Santos, A. A. P., Silva, J. M. O., \& Sanches, M. E. T. L. (2016). Assistência de enfermagem obstétrica baseada em boas práticas: do acolhimento ao parto. Revista Eletrônica De Enfermagem, 18:e1166. http://doi.org/10.5216/ree.v18.36714

Vogt, S. E., Silva, K. S., \& Dias, M. A. B. (2014). Comparação de modelos de assistência ao parto em hospitais públicos. Revista de Saúde Pública, 48(2), 304-313. http://doi.org/10.1590/S0034-8910.2014048004633

World Health Organization. (2018). WHO recommendations: intrapartum care for a positive childbirth experience [Internet]. Geneva: WHO; http://apps.who.int/iris/bitstream/10665/260178/1/9789241550215-eng.pdf?ua=1 